\title{
Lutein inhibits proliferation, invasion and migration of hypoxic breast cancer cells via downregulation of HES1
}

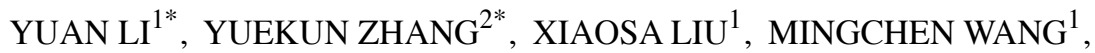 \\ PENG WANG $^{3}$, JIANBO YANG ${ }^{4}$ and SHANFENG ZHANG ${ }^{1}$ \\ ${ }^{1}$ Department of Biochemistry and Molecular Biology, School of Basic Medical Sciences, Zhengzhou University, \\ ${ }^{2}$ School of Clinical Medicine, Zhengzhou University; ${ }^{3}$ Department of Basic Medicine, Nursing College, \\ Zhengzhou University, Zhengzhou, Henan 450001, P.R. China; ${ }^{4}$ Medical Laboratories, \\ University of Virginia Health System, Charlottesville, VA 22903, USA
}

Received November 22, 2017; Accepted March 14, 2018

DOI: $10.3892 /$ ijo.2018.4332

\begin{abstract}
An intratumoral hypoxic microenvironment is frequently observed in solid tumors, including breast cancer. Lutein, a plant-derived compound and non-vitamin A carotenoid, has been demonstrated to possess multiple protective properties including anti-inflammation, anti-oxidative stress and antitumor effects. The main objective of the present research was to elucidate the involvement of lutein in the production of reactive oxygen species (ROS) under hypoxia, the activation of hairy and enhancer of split 1 (HES1), and the proliferation, invasion and migration of breast cancer cells. The human breast cancer cell lines MDA-MB-157 and MCF-7 were exposed to hypoxic conditions and various concentrations of lutein. An MTT [3-(4,5-dimethylthiazol-2-yl)-2,5-diphenyltetrazolium bromide] assay was performed to examine cell proliferation, and Annexin V-fluorescein isothiocyanate/propidium iodide staining was performed to analyze the apoptosis ratio. The levels of hypoxia inducible factor- $1 \alpha$ (HIF-1 $\alpha)$, NOTCH signaling molecules, HES1 and epithelial-mesenchymal transition (EMT)-associated factors were examined by reverse transcription-quantitative polymerase chain reaction and western blot analysis. Wound healing and Transwell invasion assays were used to detect the invasion and migration of breast cancer cells. Intracellular ROS levels were examined using 2,7-dichlorodihydrofluorescein-diacetate and flow cytometry.
\end{abstract}

Correspondence to: Dr Shanfeng Zhang, Department of Biochemistry and Molecular Biology, School of Basic Medical Sciences, Zhengzhou University, 100 Kexue Avenue, Zhengzhou, Henan 450001, P.R. China

E-mail: zsf@zzu.edu.cn

${ }^{*}$ Contributed equally

Abbreviations: LUT, lutein; EMT, epithelial-mesenchymal transition; ROS, reactive oxygen species; NAC, N-acetylcysteine

Key words: lutein, hypoxia, reactive oxygen species, hairy and enhancer of split 1 , breast neoplasms
The results revealed that cell proliferation was inhibited by lutein in a dose-dependent manner, and the apoptosis ratio gradually increased with lutein treatment under hypoxia as evident from flow cytometry-based analysis. Exposure to lutein inhibited hypoxia-mediated activation of HIF-1 $\alpha$, NOTCH signaling and HES1 expression, and suppressed the hypoxia-induced expression of EMT-associated factors. Lutein markedly inhibited the invasion and migration of breast cancer cells under hypoxia. Hypoxia-induced production of ROS was also decreased by lutein. Furthermore, the ROS scavenger $\mathrm{N}$-acetylcysteine also suppressed hypoxia inducible factor $1 \alpha$ and HES1 expression in breast cancer cells during hypoxia, but hydrogen peroxide $\left(\mathrm{H}_{2} \mathrm{O}_{2}\right)$ levels were increased. Taken together, the results of the present study suggested that lutein may be a novel candidate for the chemoprevention of breast cancer. Furthermore, HES1 may be crucial in mediating the involvement of lutein in the suppression of hypoxia-driven ROS-induced breast cancer progression.

\section{Introduction}

Breast cancer is one of the most prevalent malignant cancers and the second leading cause of cancer-associated mortality among women globally (1). Morbidity and mortality in breast cancer are high in developed countries and are on the increase in developing countries (2). Susceptibility to breast cancer is assessed through multiple factors, including environmental factors, physical factors and genetic factors $(1,2)$. The phenotype, prognosis and molecular hallmarks of breast cancer are heterogeneous. Considering the markers of mammary epithelial cell states, it is possible to classify breast cancer into stemcell-like, basal and luminal cancer $(3,4)$. Triple-negative breast cancer, which lacks estrogen receptors, progesterone receptors and human epidermal growth factor receptor-2 (HER-2) is the most malignant breast cancer, and effective treatments remain unavailable (5). To date, resection followed by loco-regional radiotherapy and systemic treatments constitutes primary therapy in breast cancer (6). Surgery has improved outcomes in primary breast cancer, but resistance to radiotherapy, endocrine therapy and chemotherapy, along with severe toxicity in locally advanced and metastatic cancers, limits the efficiency 
of therapies and contributes to mortality in breast cancer $(1,7)$. Therefore, novel therapeutic options for the treatment of breast cancer are urgently needed.

The center of solid tumors commonly develops a pathophysiological condition, which is characterized by hypoxia due to rapid tumor growth and limited oxygen diffusion (8). Intratumoral hypoxic conditions contribute to tumor angiogenesis, invasion, metastasis and resistance to therapies; these events are also associated with aggressive cancer phenotypes $(6,9)$. When adapting to the hypoxic microenvironment of tumors, cells activate certain hypoxia-associated proteins and signaling pathways, including hypoxia inducible factor 1 (HIF-1) (10). HIF-1, a heterodimer consisting of HIF-1 $\alpha$ and HIF-1 $\beta$, belongs to the family of basic helix-loop-helixperiodic acid-Schiff domain transcription factors. It regulates the expression of $>70$ genes, which serve pivotal functions in low oxygen metabolism, tumorigenesis, angiogenesis and metastasis (11). HIF-1 $\alpha$ functions as an oxygen-sensitive factor of HIF-1, which is hydroxylated at proline resides (pro ${ }^{402}$ and pro $^{564}$ ) of the oxygen-dependent disintegration domain by prolyl hydroxylase, and interacts with von Hippel-Lindau protein. This interaction results in ubiquitination and disintegration of HIF-1 $\alpha$ through the recruitment of the E3 ubiquitin ligase under normoxia; however, HIF-1 $\alpha$ is accumulated under hypoxia (10). In addition to oxygen-dependence, oxygenindependent mechanisms have also been demonstrated to regulate HIF-1 $\alpha$ in tumor cells under normoxia (11). Excessive accumulation of HIF- $1 \alpha$ has been observed in human tumors compared with para-carcinoma tissues, such as non-small-cell lung cancer and ovarian cancer, suggesting that it may be a potential target for neoplastic therapy (8).

The hairy and enhancer of split (HES) family has seven members, HES1-7, which belong to the basic helix-loop-helix superfamily of DNA-binding transcription factors and directly affect cellular activities (12). Studies have demonstrated that HES1 affects cell proliferation and differentiation during embryogenesis, maintenance of stem cells, and the malignancy and maintenance of tumor cells (13). Overexpression of HES1 is associated with the development of several types of cancer, including lung cancer, cervical cancer, prostate cancer, oral squamous cell carcinoma, pancreatic cancer, colon carcinoma, and ovarian cancer (14-16). The function of HES1 and its involvement in the regulation in malignant breast cancer remain to be fully elucidated; therefore, it is necessary to investigate its function and underlying mechanisms in breast cancer proliferation and metastasis. Epithelial-mesenchymal transition (EMT) is one of the crucial mechanisms that induce tumor invasion, metastasis, and migration, as it facilitates cancer dissemination. EMT is characterized by the transformation of the cellular phenotype from the epithelial phenotype (non-motile, polarized and collective) to the mesenchymal phenotype (individual, non-polarized, motile and invasive) (17). The EMT process involves dissolution of epithelial cell-cell adhesion, cytoskeletal actin remodeling and overexpression of mesenchymal adhesive molecular markers. Previous studies have suggested that hypoxia-induced invasion and the EMT processes are associated with HES1 $(10,17)$.

A number of studies have demonstrated that phytochemicals have potential for the prevention and treatment of cancer. Lutein is a natural dihydroxycarotenoid belonging to the family of non-vitamin A carotenoids, which are the second most widespread carotenoid in human serum and are present in deep yellow vegetables and fruits, including lettuce and spinach (18). Lutein has anti-oxidative, anti-inflammatory, and antitumor properties. Concentrated in the retina, lutein decreases the risk of age-associated macular degeneration due to its anti-oxidative properties (19). Previous studies have demonstrated that lutein exhibits potential antitumor properties in several cancers, including cervical carcinoma, colon cancer and hepatic carcinoma (20-22). Lutein is involved in cancer initiation and progression by regulating cellular redox status and associated signaling pathways. However, its effects on breast cancer and underlying mechanisms remain to be fully investigated.

The aim of the present study was to elaborate the molecular mechanism underlying the inhibitory effect of lutein in hypoxia-induced reactive oxygen species (ROS) production, and the proliferation, invasion and migration of breast cancer cells. Lutein was demonstrated to inhibit cell vitality in several breast cancer cell lines in a dose-dependent manner. Lutein downregulated HES1 expression by inhibiting the generation of ROS and expression of HIF-1 $\alpha$ and NOTCH. Lutein also suppressed EMT, cell invasion, and metastasis in breast cancer cells. The results of the present study suggest that the antitumor effects of lutein may enable its application in the treatment of breast cancer via the inhibition of HES1.

\section{Materials and methods}

Reagents. Lutein ( $>99 \%$ pure) was acquired commercially (Sigma-Aldrich; Merck KGaA, Darmstadt, Germany) and dissolved in dimethyl sulfoxide (DMSO). It was then added to RPMI-1640 medium (Gibco; Thermo Fisher Scientific, Inc., Waltham, MA, USA) to each working dose. N-acetylcysteine (NAC) and hydrogen peroxide $\left(\mathrm{H}_{2} \mathrm{O}_{2}\right)$ were purchased from Sigma-Aldrich; Merck KGaA. All other reagents were of reagent grade and purchased from standard commercial sources. All drug solutions were freshly compounded on the day of the test.

Cell culture and transfection. The human breast cancer cell lines, MDA-MB-157 and MCF-7, were obtained from the Type Culture Collection of the Chinese Academy of Sciences (Shanghai, China). Short tandem repeat profiling analysis was used to examine and authenticate breast cell lines, and they were used within 6 months of authentication. Cells were grown in RPMI-1640 medium-supplemented with $10 \%$ fetal bovine serum (Gibco; Thermo Fisher Scientific, Inc.), $100 \mathrm{U} / \mathrm{ml}$ penicillin, and $100 \mu \mathrm{g} / \mathrm{ml}$ streptomycin. Cells were cultured under normoxia in a humidified atmosphere with $5 \% \mathrm{CO}_{2}$ at $37^{\circ} \mathrm{C}$. The cells cultured under hypoxia were placed in a hypoxic chamber (Thermo Fisher Scientific, Inc.) suffused with $1 \% \mathrm{O}_{2}$, $5 \% \mathrm{CO}_{2}$, and $94 \% \mathrm{~N}_{2}$ at $37^{\circ} \mathrm{C}$.

The human HES1 expression vector HES1-pCMV6-XL5 was obtained from OriGene Technologies, Inc. (Rockville, MD, USA). An empty vector was employed as a negative control. MDA-MB-157 and MCF-7 cells were transiently transfected with HES1 overexpression constructs. When these cells reached $60-80 \%$ confluence, they were transfected with the appropriate constructs using Lipofectamine ${ }^{\mathrm{TM}} 2000$ transfection reagent (Invitrogen; Thermo Fisher Scientific, Inc.) according to the 
Table I. Primer sequences used for reverse transcription-quantitative polymerase chain reaction analysis.

\begin{tabular}{lll}
\hline Gene name & \multicolumn{1}{c}{ Forward primer sequence $\left(5^{\prime}-3^{\prime}\right)$} & \multicolumn{1}{c}{ Reverse primer sequence $\left(5^{\prime}-3^{\prime}\right)$} \\
\hline HES 1 & TCAACACGACACCGGATAAA & CCGCGAGCTATCTTTCTTCA \\
E-cadherin & CAGCACGTACACAGCCCTAA & ACCTGAGGCTTTGGATTCCT \\
N-cadherin & ACAGTGGCCACCTACAAAGG & CCGAGATGGGGTTGATAATG \\
Vimentin & GAGAACTTTGCCGTTGAAGC & TCCAGCAGCTTCCTGTAGGT \\
HIF-1 $\alpha$ & GTCGGACAGCCTCACCAAACAGAGC & GTTAACTTGATCCAAAGCTCTGAG \\
NOTCH3 & TCTTGCTGCTGGTCATTCTC & TGCCTCATCCTCTTCAGTTG \\
JAG1 & GACTCATCAGCCGTGTCTCA & TGGGGAACACTCACACTCAA \\
JAG2 & TCTGCCTTGCTACAATGGTG & GCGATACCCGTTGATCTCAT \\
DLL1 & TGCAACCCTGGCTGGAAA & AATCCATGCTGCTCATCACATC \\
$\beta$-actin & TCCCTGGAGAAGAGCTACGA & ACCTGAGGCTTTGGATTCCT
\end{tabular}

HES1, hairy and enhancer of split 1; E-cadherin, epithelial-cadherin; N-cadherin, neural-cadherin; HIF-1 $\alpha$, hypoxia inducible factors-1 $\alpha$; JAG, jagged; DLL1, delta-like 1 .

manufacturer's protocol. After $24 \mathrm{~h}$, transfection efficiency was checked by reverse transcription-quantitative polymerase chain reaction (RT-qPCR) and western blot analysis. Stably transfected cells were used for subsequent experiments.

Cell proliferation assay. MDA-MB-157 and MCF-7 cells were plated onto 96-well culture plates with various concentrations $(5,10,20,40,80$, and $120 \mu \mathrm{M})$ of lutein; $0 \mu \mathrm{M}$ lutein was used as negative control. Cells were incubated for $24 \mathrm{~h}$ in a humidified incubator with $5 \% \mathrm{CO}_{2}$ at $37^{\circ} \mathrm{C}$. At the end of the treatment, cell viability was examined with the MTT assay. MTT reagent (50 $\mu 1$, Sigma-Aldrich; Merck KGaA) was added to each well, and the plate was incubated for another $2 \mathrm{~h}$ at $37^{\circ} \mathrm{C}$. Formazan, which is produced from MTT by the action of viable cells, was dissolved in $100 \mu \mathrm{l}$ DMSO. Absorbance at $570 \mathrm{~nm}$ was measured using a microplate reader (Infinite M200; Tecan Group, Ltd., Mannedorf, Switzerland). The concentration of lutein required for a $50 \%$ reduction in cell viability (concentration of an inhibitor where the response/ binding is reduced by half) was calculated and used in further experiments. The half maximal inhibitory concentration $\left(\mathrm{IC}_{50}\right)$ values were calculated from the percentage of cell viability at each concentration of lutein, using probit analysis.

Cell invasion assay. Invasion assays of breast cancer cells were performed using Transwell chambers (Corning Incorporated, Corning, NY, USA) with an 8-micrometer pore insert pre-coated with Matrigel in a 24-well plate (Corning Incorporated). Cell suspension $\left(5 \times 10^{4}\right.$ cells $\left./ \mathrm{ml}\right)$ in serum-free medium was added to the upper chambers, and $600 \mu \mathrm{l}$ medium containing $10 \%$ fetal bovine serum was added to the lower chambers. Next, the chamber was incubated under hypoxia for $24 \mathrm{~h}$ or treated with lutein $(40 \mu \mathrm{M})$ under hypoxia at $37^{\circ} \mathrm{C}$ for $24 \mathrm{~h}$. A normoxic control was included to detect the effects of lutein on cell invasion. Following incubation, the cells in the upper chambers were gently scraped off using a cotton-tipped swab. Invading cells were fixed with $5 \%$ paraformaldehyde for $30 \mathrm{~min}$ and stained with $0.1 \%$ crystal violet for $15 \mathrm{~min}$ at room temperature in the lower chambers. Cell count on the lower side of the membrane was obtained as the average of the cell counts from five random fields in each well, which was captured at x200 magnification under a bright-field microscope.

Wound healing assay. The wound healing assay was performed to detect the migratory ability of cancer cells. Breast cancer cells were seeded into 6-well plates to reach a confluent monolayer. Once the cells reached $90 \%$ confluence, monolayers were scratched with a 200-microliter microtip to produce a wound line among the cells. Cells were washed with PBS twice and then covered with serum-free medium either in the absence of stimulus or in the presence of lutein $(40 \mu \mathrm{M})$. Next, cells were allowed to migrate for $24 \mathrm{~h}$ at $37^{\circ} \mathrm{C}$ under normoxia or hypoxia. Cell migration images were captured at 0 and $24 \mathrm{~h}$ under a Leica DMIL inverted microscope (x50 magnification; Leica Microsystems GmbH, Wetzlar, Germany) from three independent experiments. The edge from 0 to $24 \mathrm{~h}$ was assessed using ImageJ software (National Center for Biotechnology Information, Bethesda, MD, USA) to calculate the relative distance travelled.

Cell apoptosis assays. The Annexin V-fluorescein isothiocyanate (FITC)/propidium iodide (PI) apoptosis detection kit was obtained from Beyotime Institute of Biotechnology (Haimen, China). The content of apoptotic cells was estimated following the manufacturer's protocol. Cells were exposed to the indicated concentrations $(20,40$, and $80 \mu \mathrm{M})$ of lutein and incubated for $24 \mathrm{~h}$ at $37^{\circ} \mathrm{C}$ under normoxia or hypoxia. Once they were harvested from the plates, cells were washed and suspended in PBS. Next, cells were stained with Annexin V-FITC and PI. The percentage of apoptotic cells was detected using flow cytometry (BD Biosciences, San Jose, CA, USA) and the data were analyzed using CellQuest software (version 5.1; BD Biosciences). A total of $1 \times 10^{4}$ events were collected for each run, and the assay was repeated in three independent replicates.

ROS detection. ROS levels were detected using the 2,7-dichlorodihydrofluorescein-diacetate (DCFH-DA) Cellular ROS Detection assay kit (Beyotime Institute of Biotechnology), according to the manufacturer's protocol. In brief, breast cancer 
cells were seeded in 6-well plates and exposed to normoxia or hypoxia or subjected to treatment with lutein $(40 \mu \mathrm{M})$, $\mathrm{H}_{2} \mathrm{O}_{2}(200 \mu \mathrm{M})$ or N-acetylcysteine (NAC; $\left.50 \mu \mathrm{M}\right)$ for $24 \mathrm{~h}$ at $37^{\circ} \mathrm{C}$. Cells were $\left(1 \times 10^{6}\right.$ cells $\left./ \mathrm{ml}\right)$ trypsinized and suspended in DCFH-DA $(10 \mu \mathrm{M})$ for $20 \mathrm{~min}$ at $37^{\circ} \mathrm{C}$ then washed with PBS twice. Intracellular ROS levels were measured in each group according to DCF fluorescence by flow cytometry (BD Biosciences); data were analyzed using CellQuest 5.1 software (BD Biosciences).

$R N A$ extraction and $R T-q P C R$. Total RNA was isolated from breast cancer cells using TRIzol reagent (Invitrogen; Thermo Fisher Scientific, Inc.). The concentration of total RNA was measured using a Nanodrop 2000C spectrophotometer (Thermo Fisher Scientific, Inc.), and reverse transcription of total RNA was performed using the PrimeScript RT Reagent kit (Takara Biotechnology Co., Ltd., Dalian, China) according to the manufacturer's protocol. qPCR was performed using the SYBR Green PCR kit (Takara Biotechnology Co., Ltd.) on an Applied Biosystems 7500 fast Real-Time PCR System (Thermo Fisher Scientific, Inc.). The following PCR reaction conditions were used: $95^{\circ} \mathrm{C}$ for $30 \mathrm{sec}$, followed by 40 cycles consisting of $95^{\circ} \mathrm{C}$ for $5 \mathrm{sec}$ and $60^{\circ} \mathrm{C}$ for $30 \mathrm{sec}$. Melting curve analysis was performed from $60^{\circ} \mathrm{C}$ to $95^{\circ} \mathrm{C}$ to assess the specificity of PCR products. All RT-qPCR primer sequences are listed in Table I. The expression level of each gene was evaluated using the comparative $\mathrm{Cq}$ method (23), and $\beta$-actin mRNA levels were used as a normalization control.

Western blot analysis. Cells were lysed in RIPA lysis buffer (Beyotime Institute of Biotechnology) supplemented with $1 \mathrm{mM}$ PMSF (Beyotime Institute of Biotechnology). This lysate was centrifuged at $12,000 \mathrm{x}$ g for $15 \mathrm{~min}$ at $4^{\circ} \mathrm{C}$ and supernatant was harvested. Protein concentration was measured using BCA reagent (Thermo Fisher Scientific, Inc.). Protein extracts $(50 \mu \mathrm{g})$ underwent $10 \%$ SDS-PAGE and transferred to a nitrocellulose membrane (Merck KGaA). These membranes were soaked in $5 \%$ non-fat dry milk for $2 \mathrm{~h}$ at $4^{\circ} \mathrm{C}$ and incubated with primary antibodies against HIF-1 $\alpha$ (1:800, ab113642; Abcam, Cambridge, UK), HES1 (1:500, ab71559; Abcam), NOTCH3 (1:800, sc515825; Santa Cruz Biotechnology, Inc., Dallas, TX, USA), JAGGED1 (JAG1; 1:500, \#70109; Cell Signaling Technology, Inc., Danvers, MA, USA), JAGGED2 (JAG2; 1:500, \#2210; Cell Signaling Technology, Inc.), Delta-like 1 (DLL1; 1:800, MAB1818; R\&D Systems, Inc., Minneapolis, MN, USA), epithelial-cadherin (E-cadherin; 1:800, ab15148; Abcam), neural-cadherin (N-cadherin; 1:800, ab98952; Abcam), vimentin (1:800, \#5741; Cell Signaling Technology, Inc.), and $\beta$-actin (1:1000, ab227387; Abcam) overnight at $4^{\circ} \mathrm{C}$. Next, membranes were incubated with horseradish peroxidaseconjugated secondary goat anti-mouse (1:2000, HAF007; R\&D Systems, Inc.) or goat anti-rabbit IgG antibodies (1:2000, HAF008; R\&D Systems, Inc.) at $37^{\circ} \mathrm{C}$ for $2 \mathrm{~h}$. Immunoreactive protein bands were visualized using a chemiluminescence assay (Beyotime Institute of Biotechnology).

Immunofluorescence analyses. Following the indicated treatments, breast cancer cells were rinsed with PBS twice, fixed in fresh $4 \%$ paraformaldehyde for $30 \mathrm{~min}$ at $37^{\circ} \mathrm{C}$, and permeabilized with $0.2 \%$ Triton X-100 (Sigma-Aldrich; Merck
KGaA). Cells were blocked with 5\% BSA (Sigma-Aldrich; Merck KGaA) in PBS at $37^{\circ} \mathrm{C}$ for 30 min and incubated overnight with primary antibodies against $\mathrm{E}$-cadherin, $\mathrm{N}$-cadherin and vimentin (all diluted 1:100) at $4^{\circ} \mathrm{C}$. It was followed by incubation with secondary FITC-conjugated goat anti-rabbit IgG antibody (1:200, AP307F; Chemicon International, Inc., Temecula, CA, USA) or tetramethylrhodamine-conjugated goat anti-mouse IgG antibody (1:200, AP500R; Chemicon International, Inc.) for $30 \mathrm{~min}$ at room temperature, and then counterstained with DAPI prior to observation. The target protein appeared green and the nuclei appeared blue, as visualized by a fluorescence microscope (Olympus BX43; Olympus Corporation, Tokyo, Japan) at x200 magnification. Image-Pro Plus 6.0 software (Media Cybernetics, Inc., Rockville, MD, USA) was used to process the images.

Statistical analysis. All data were analyzed using SPSS 21.0 (IBM Corp., Armonk, NY, USA) or GraphPad Prism version 5.0 (GraphPad Software, Inc., La Jolla, CA, USA). Data were obtained from at least three independent experiments and presented as mean \pm standard deviation. One-way ANOVA and Dunnett's test was used for the comparison of means between groups. Unpaired Student's t-tests were used to compare between two groups. $\mathrm{P}<0.05$ was considered to indicate a statistically significant difference.

\section{Results}

Lutein suppresses the viability and induces the apoptosis of breast cancer cells under normoxia and hypoxia. To determine whether lutein affects cell viability, MDA-MB-157 and MCF-7 human breast cancer cells were treated with 5-120 $\mu \mathrm{M}$ lutein for $24 \mathrm{~h}$ under normoxia and hypoxia. As presented in Fig. 1A and B, lutein markedly decreased cell viability in a concentration-dependent manner under normoxia and hypoxia. Hypoxia alone induced a lower inhibitory rate with lutein intervention than normoxia did in MDA-MB-157 and MCF-7 cells, and low oxygen exposure improved the $\mathrm{IC}_{50}$ of lutein in breast cancer cells (Fig. 1C). The Annexin V-PI assay was used to detect the extent of apoptosis induced by lutein in MDA-MB-157 and MCF-7 cells (Fig. 1D). Apoptosis was increased in cells treated with lutein compared with the untreated control. Similarly, hypoxia, compared with normoxia, inhibited cell apoptosis. These results suggested that lutein treatment inhibited the viability and promoted the apoptosis of MDA-MB-157 and MCF-7 cells under normoxia and hypoxia. Furthermore, hypoxia increased the viability of cancer cells compared with normoxia.

Antitumor effects of lutein are mediated through downregulation of HES1. HES1 is involved in the regulation of cell cycle, growth, differentiation, invasion, and apoptosis in various cancers $(12,13)$. Therefore, the involvement of HES1 in the effects of lutein on tumor inhibition were investigated. The results revealed that mRNA and protein levels of HES1 were significantly upregulated under hypoxia compared with normoxia, and lutein intervention decreased the expression of HES1 (Fig. 2). To investigate whether downregulation of HES1 was a mechanism underlying the antitumor effects of lutein, HES1 mRNA and protein was transiently overexpressed in 
A

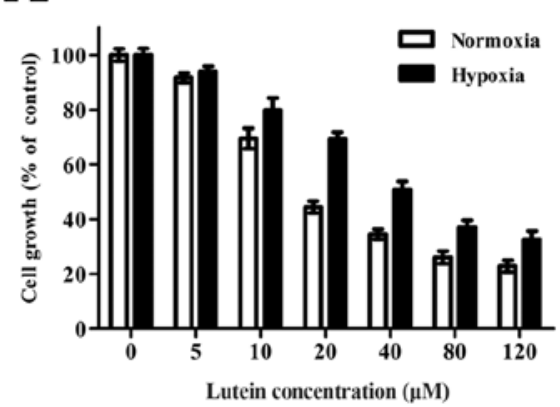

B

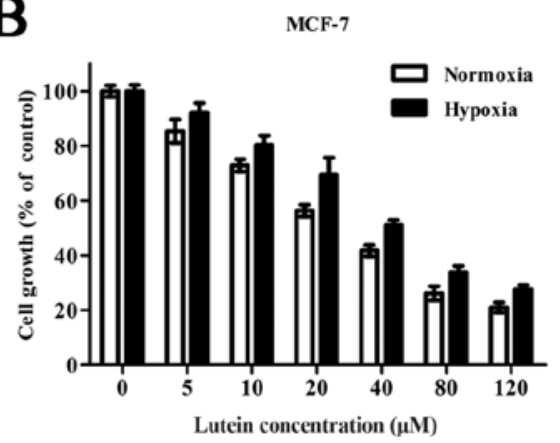

C

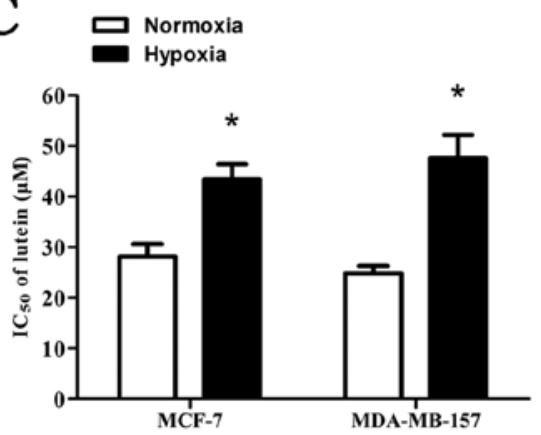

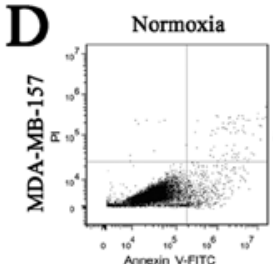

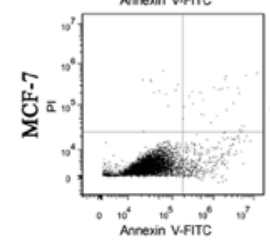

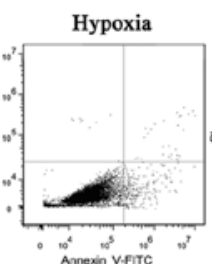
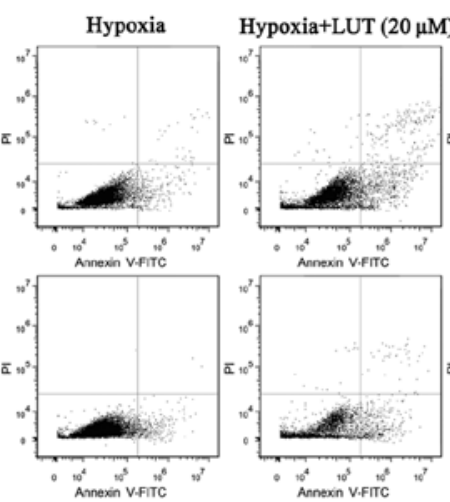
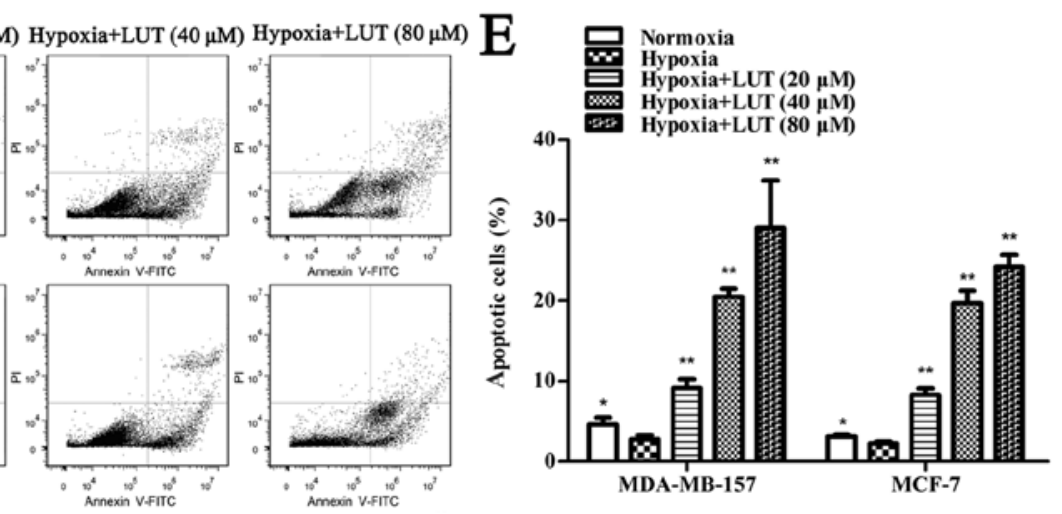

Figure 1. LUT inhibited cell viability and induced apoptosis in breast cancer cells under normoxia and hypoxia. Cell viability in (A) MDA-MB-157 and (B) MCF-7 cells was measured after $24 \mathrm{~h}$ of treatment with increasing concentrations of LUT under normoxia or hypoxia. (C) Comparison of IC $\mathrm{C}_{50}$ values of MDA-MB-157 and MCF-7 cells following LUT treatment under normoxia or hypoxia, according to the MTT assay. ${ }^{*} \mathrm{P}<0.05$ and ${ }^{* * *} \mathrm{P}<0.01$ compared with the normoxia group. (D) MDA-MB-157 and MCF-7 cells were treated with increasing concentrations of LUT under normoxia or hypoxia for $24 \mathrm{~h}$ and apoptosis was analyzed using flow cytometry, with (E) quantification. ${ }^{*} \mathrm{P}<0.05$ and $^{* *} \mathrm{P}<0.01$ compared with the hypoxia group. Double-staining with Annexin V-FITC and PI was performed to detect the ratio of apoptotic cells. The results were presented as the mean \pm standard deviation of triplicate experiments. LUT, lutein; $\mathrm{IC}_{50}$, half maximal inhibitory concentration; FITC, fluorescein isothiocyanate; PI, propidium iodide.

A

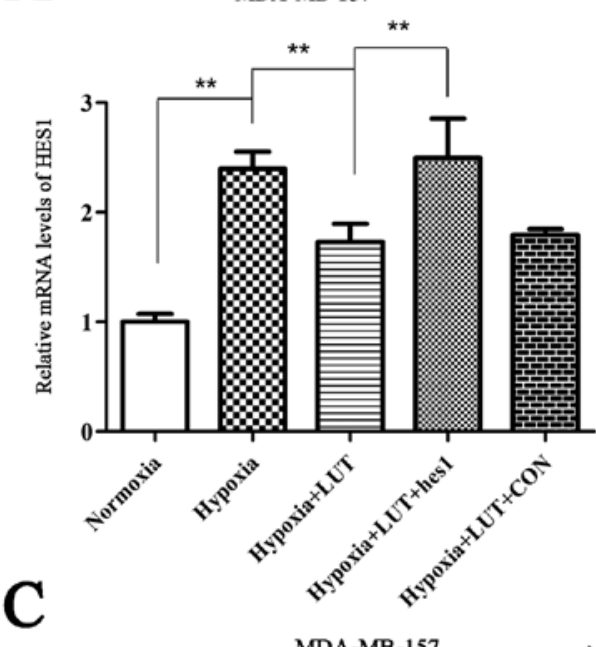

B

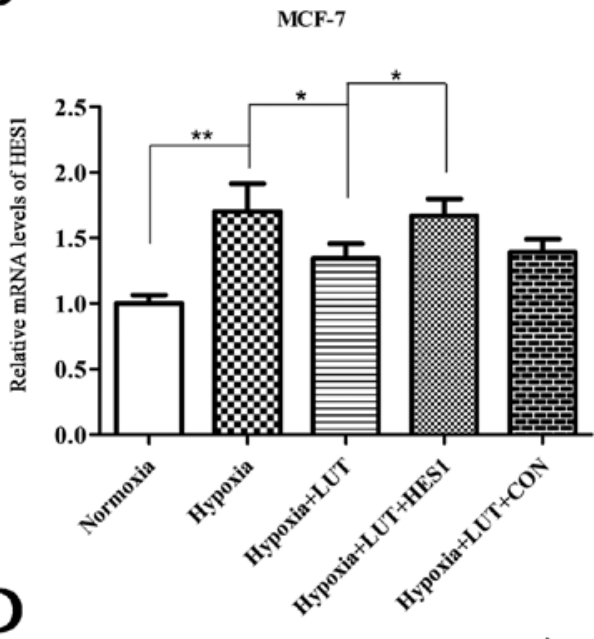

D

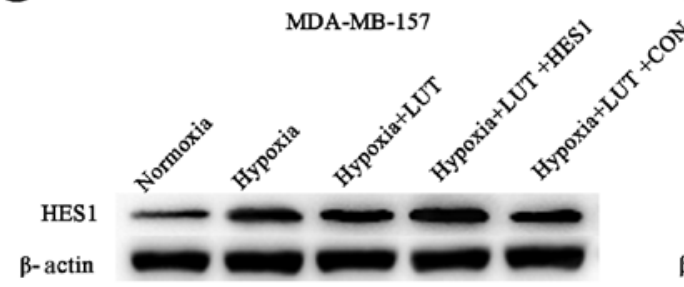

co

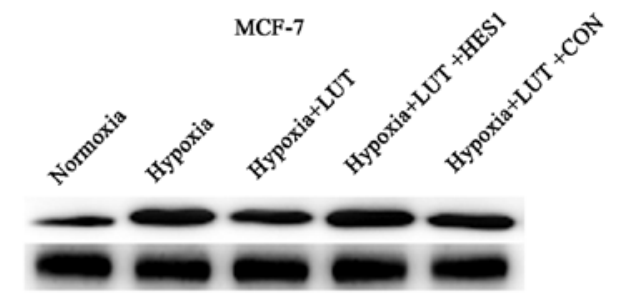

Figure 2. LUT mediates its antitumor effects through downregulation of HES1. Reverse transcription-quantitative polymerase chain reaction analysis demonstrating the effects of Lut $(40 \mu \mathrm{M})$ on hypoxia-induced mRNA expression of HES1 in (A) MDA-MB-157 and (B) MCF-7 cells following transient transfection with HES1 overexpression vectors or empty vectors. Western blotting demonstrating the effects of LUT $(40 \mu \mathrm{M})$ on hypoxia-induced protein levels of HES1 in (C) MDA-MB-157 and (D) MCF-7 cells following transient transfection with HES1 overexpression vectors or empty vectors. The results were presented as the mean \pm standard deviation of triplicate experiments. ${ }^{*} \mathrm{P}<0.05$ and ${ }^{* *} \mathrm{P}<0.01$, with comparisons indicated by lines. LUT, lutein; HES1, hairy and enhancer of split 1 . 
A

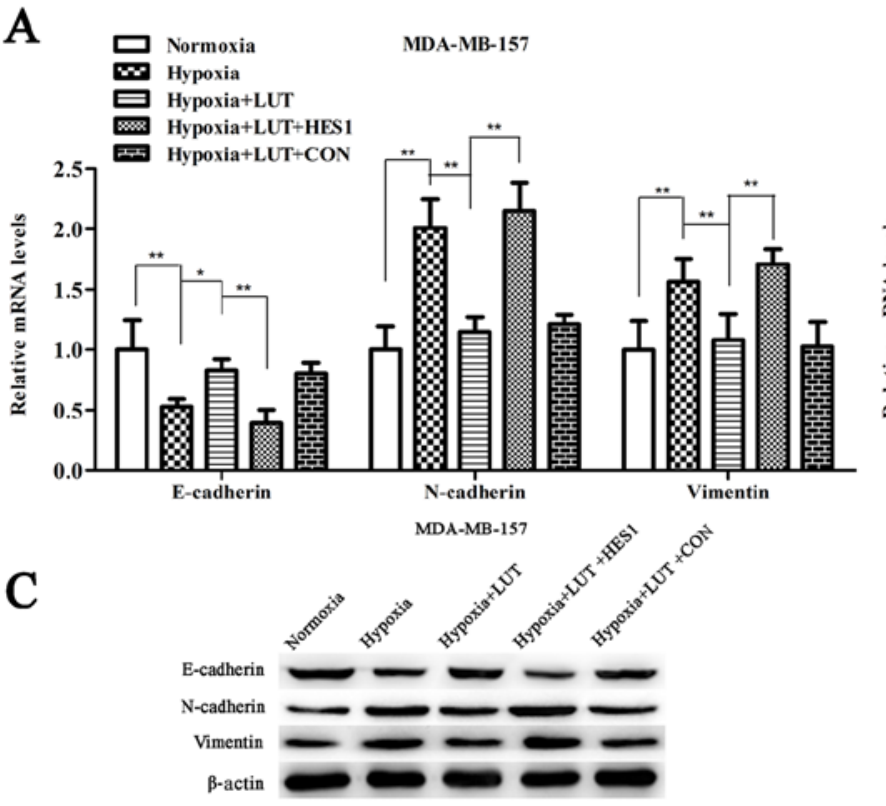

B

$$
\begin{array}{lll}
\text { B } & \text { Normoxia } & \text { MCF-7 } \\
\text { Hypoxia } & \text { Hypoxia+LUT } \\
\text { Hypoxia+LUT+HES1 } & \\
\text { Hypoxia+LUT+CON } &
\end{array}
$$

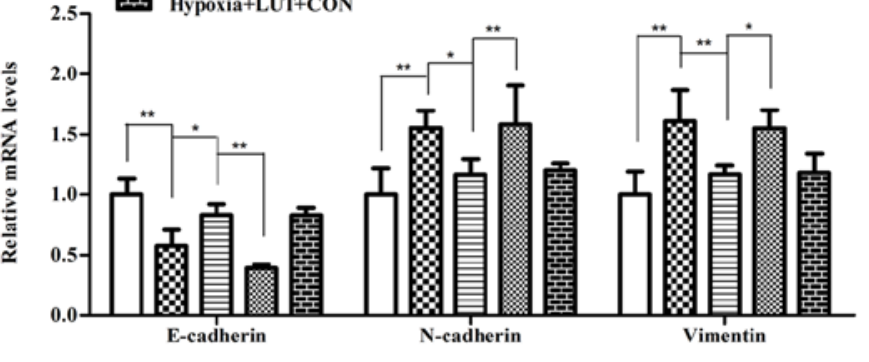

D

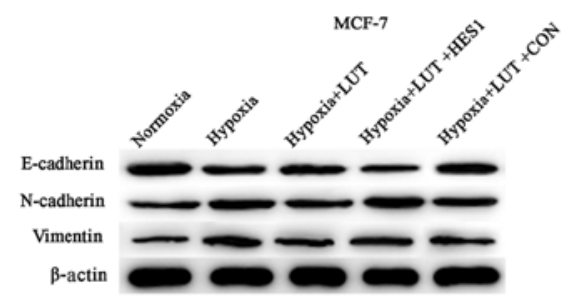

$\mathrm{E}$

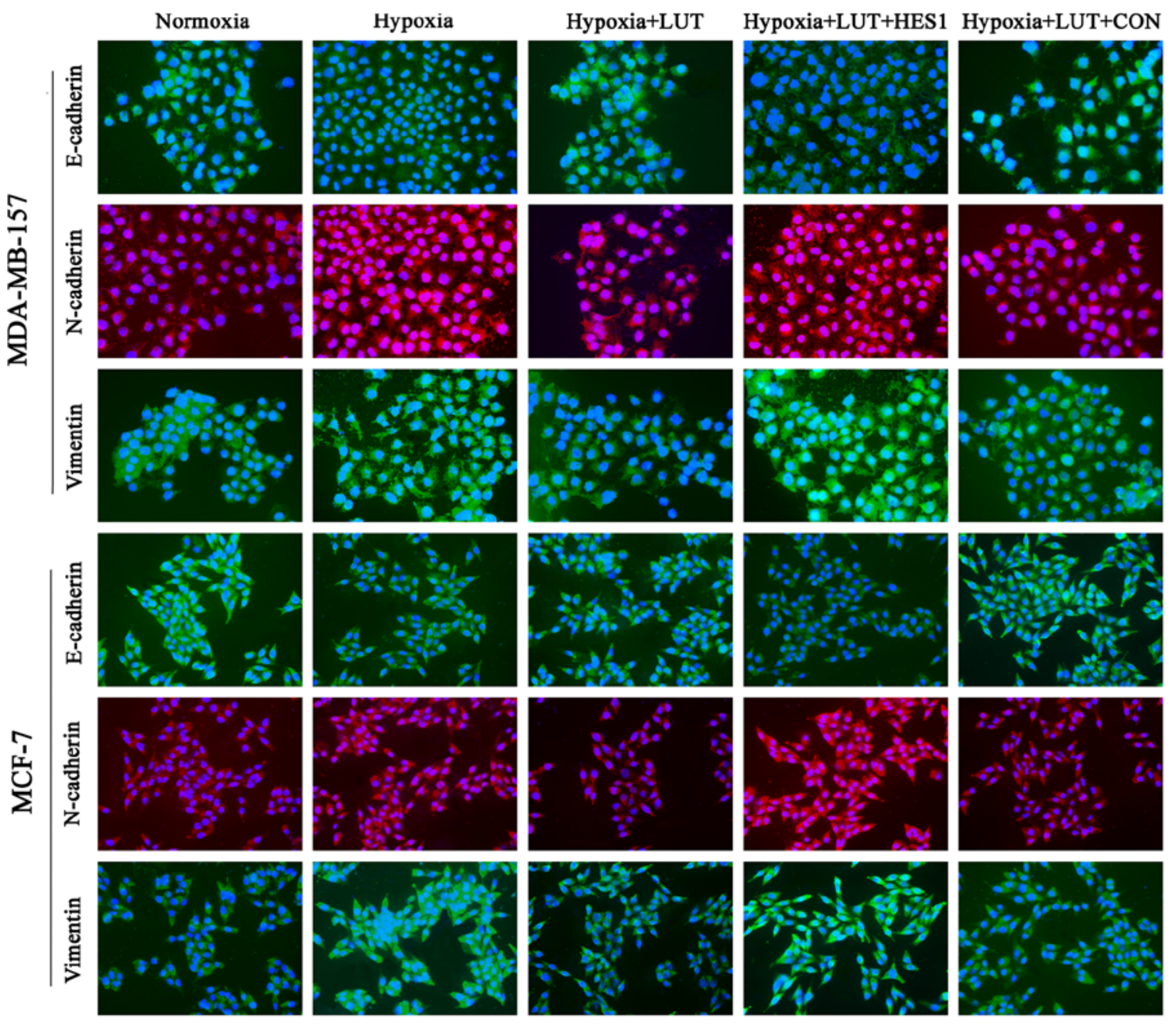

Figure 3. LUT inhibits hypoxia-induced EMT of breast cancer cells. Reverse transcription-quantitative polymerase chain reaction analysis of mRNA expression of EMT-associated genes (E-cadherin, N-cadherin, and vimentin) in (A) MDA-MB-157 or (B) MCF-7 cells stimulated with LUT (40 $\mu$ M) and subsequently transiently transfected with HES1 overexpression vectors or empty vectors under normoxia or hypoxia. Western blot analysis of EMTassociated proteins (E-cadherin, N-cadherin, and vimentin) in (C) MDA-MB-157 or (D) MCF-7 cells stimulated with LUT (40 $\mu$ M) and subsequently transiently transfected with HES1 overexpression vector or empty vector under normoxia or hypoxia. (E) MDA-MB-157 and MCF-7 cells were stimulated with LUT $(40 \mu \mathrm{M})$ and subsequently transiently transfected with HES1 overexpression vectors or empty vectors under normoxia or hypoxia as indicated. Expression levels of EMT-associated proteins (E-cadherin, N-cadherin, and vimentin) was visualized by immunofluorescence (x200 magnification). Green fluorescence indicates E-cadherin or vimentin expression. Red fluorescence indicates N-cadherin expression, and blue denotes nuclear DNA staining by DAPI. The results were presented as the mean \pm standard deviation of triplicate experiments. ${ }^{*} \mathrm{P}<0.05$ and ${ }^{* *} \mathrm{P}<0.01$, with comparisons indicated by lines. LUT, lutein; EMT, epithelial-mesenchymal transition; HES1, hairy and enhancer of split 1; E-cadherin, epithelial cadherin; N-cadherin, neural cadherin. 

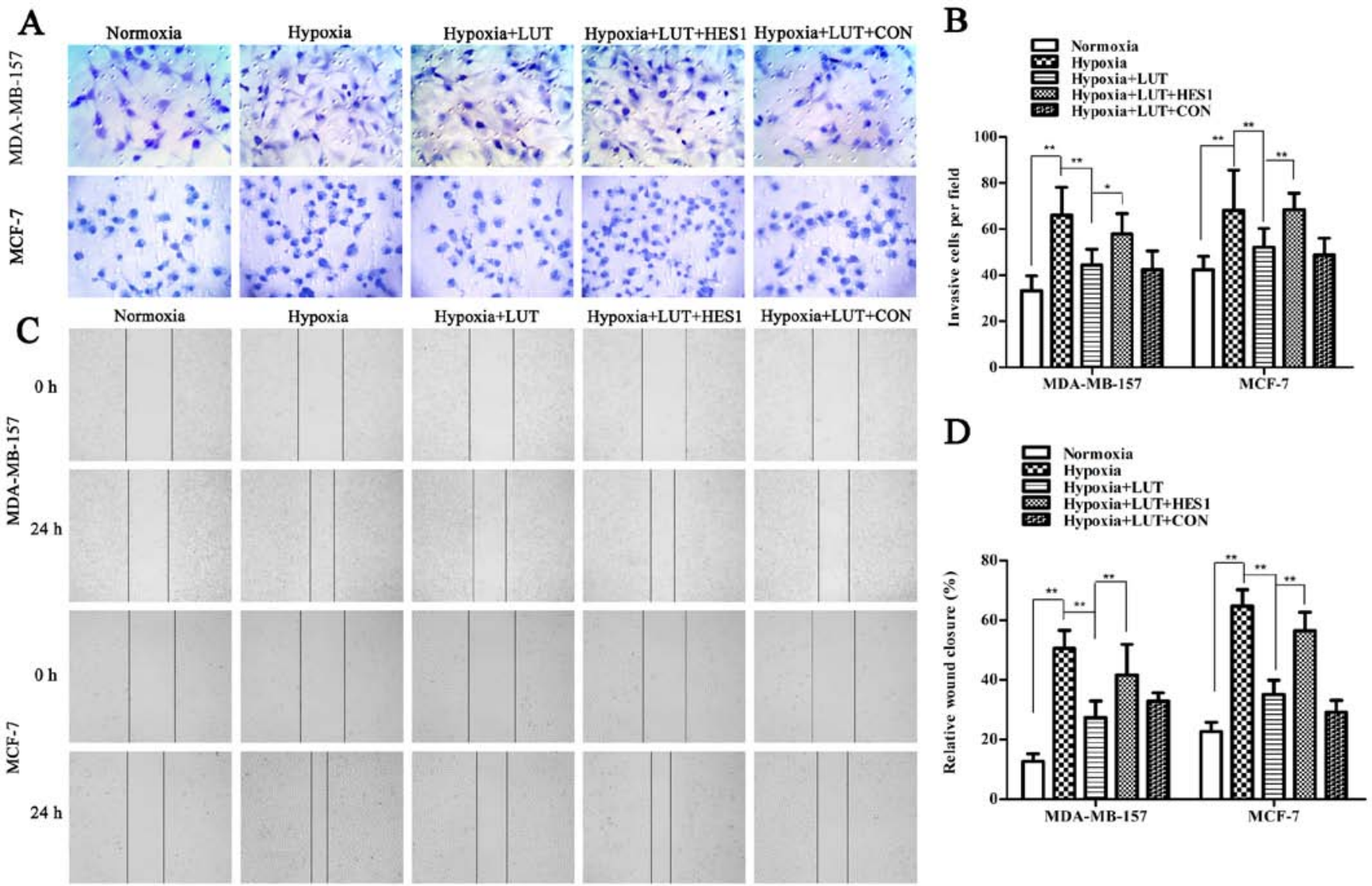

Figure 4. LUT inhibits cellular invasion and migration through inhibition of the epithelial-mesenchymal transition process. (A) Invasion was measured by Transwell invasion assays in MDA-MB-157 and MCF-7 cells stimulated with LUT $(40 \mu \mathrm{M})$ and subsequently transiently transfected with HES1 overexpression vectors or empty vectors under normoxia or hypoxia (x200 magnification), with (B) quantification. (C) Migration was measured by wound healing analysis in MDA-MB-157 and MCF-7 cells stimulated with LUT $(40 \mu \mathrm{M})$ and subsequently transiently transfected with HES1 overexpression vectors or empty vectors under normoxia or hypoxia (x40 magnification). (D) Migrated cells in the migration assays were measured and normalized to those at $0 \mathrm{~h}$. The results were presented as the mean \pm standard deviation of triplicate experiments. " $\mathrm{P}<0.05$ and ${ }^{* *} \mathrm{P}<0.01$, with comparisons indicated by lines. LUT, lutein; HES1, hairy and enhancer of split 1 .

lutein-treated MDA-MB-157 and MCF-7 cells using HES1 overexpression vectors (Fig. 2). These results suggested that the antitumor effects of lutein were associated with a decrease in HES1 expression, which was reversed by overexpression of HES1.

Lutein inhibits hypoxia-induced EMT in breast cancer cells. EMT is considered a key phenomenon that promotes tumor invasion and metastasis. Expression of HES1 is sufficient to promote EMT along with the invasive and metastatic potential of cancer cells $(12,17)$. As presented in Fig 3A, RT-qPCR and western blotting revealed that treatment with lutein significantly increased the expression of epithelial markers (E-cadherin) and abrogated the expression of mesenchymal markers (vimentin and N-cadherin) in MDA-MB-157 and MCF-7 cells (Fig. 3A-D). The changes in the expression of the EMT phenotype due to lutein interference in MDA-MB-157 and MCF-7 cell lines were further confirmed using immunocytochemistry (Fig. 3E). Next, the effects of lutein on cell invasion and motility were also investigated in breast cancer cells under hypoxia, using Transwell and wound healing assays (Fig. 4). The Transwell assay indicated that the invasiveness of MDA-MB-157 and MCF-7 cells under hypoxia was significantly inhibited by lutein treatment (Fig. 4A and B).
The spread of lutein-treated MDA-MB-157 and MCF-7 cells along the wound edges was significantly slower compared with control cells (Fig. 4C and D). This indicated that lutein significantly decreased the invasion and migration abilities of cells compared with those of control cells under hypoxia. Lutein-induced inhibition of EMT was significantly reversed by overexpression of HES1 (Fig. 3A-D). Furthermore, the lutein-induced decrease in invasion and migration was notably inhibited by overexpression of HES1 (Fig. 4). Taken together, these results indicated that lutein effectively suppressed the induction of EMT and prevented the accompanying increase in invasiveness and metastasis of breast cancer cells by downregulating HES1.

Lutein suppresses the expression of HIF-1 $\alpha$ and downstream NOTCH signaling pathway. Under hypoxia, activation of HIF- $1 \alpha$ potentiates NOTCH signaling by upregulating the expression of NOTCH receptors and ligands, which in turn induces the expression of the NOTCH target gene HES1 $(24,25)$. To determine whether the effect of lutein on HES1 expression was mediated by HIF-1 $\alpha$ transcription factor through the NOTCH signaling pathway, the expression level of HIF-1 $\alpha$, NOTCH3 and NOTCH ligands (JAG1, JAG2, and DLL1) was examined. As presented in Fig. 5, hypoxia 

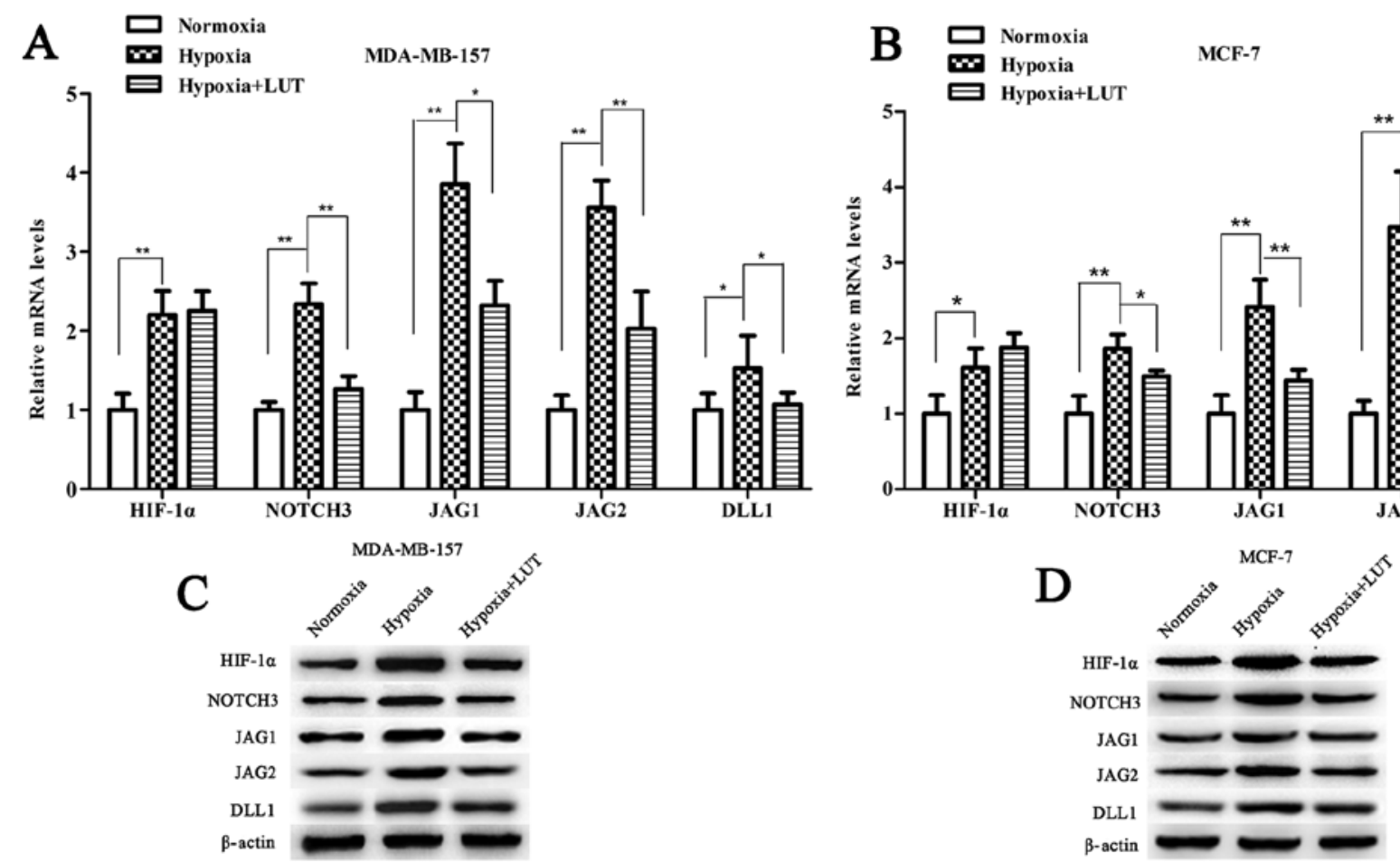

Figure 5. LUT suppresses the expression of HIF-1 $\alpha$ and the downstream NOTCH signaling pathway. Reverse transcription-quantitative polymerase chain

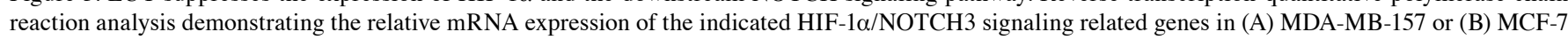
cells with or without LUT $(40 \mu \mathrm{M})$ treatment under normoxia or hypoxia for $24 \mathrm{~h}$. Western blot analysis of the expression of the indicated HIF-1 $\alpha / \mathrm{NOTCH} 3$ signaling proteins in (C) MDA-MB-157 or (D) MCF-7 cells with or without LUT $(40 \mu \mathrm{M})$ treatment under normoxia or hypoxia for $24 \mathrm{~h}$. The results were presented as the mean \pm standard deviation of triplicate experiments. ${ }^{*} \mathrm{P}<0.05$ and ${ }^{* *} \mathrm{P}<0.01$, with comparisons indicated by lines. LUT, lutein; HIF-1 $\alpha$, hypoxia inducible factor $1 \alpha$; JAG, JAGGED; DLL1, delta-like 1.
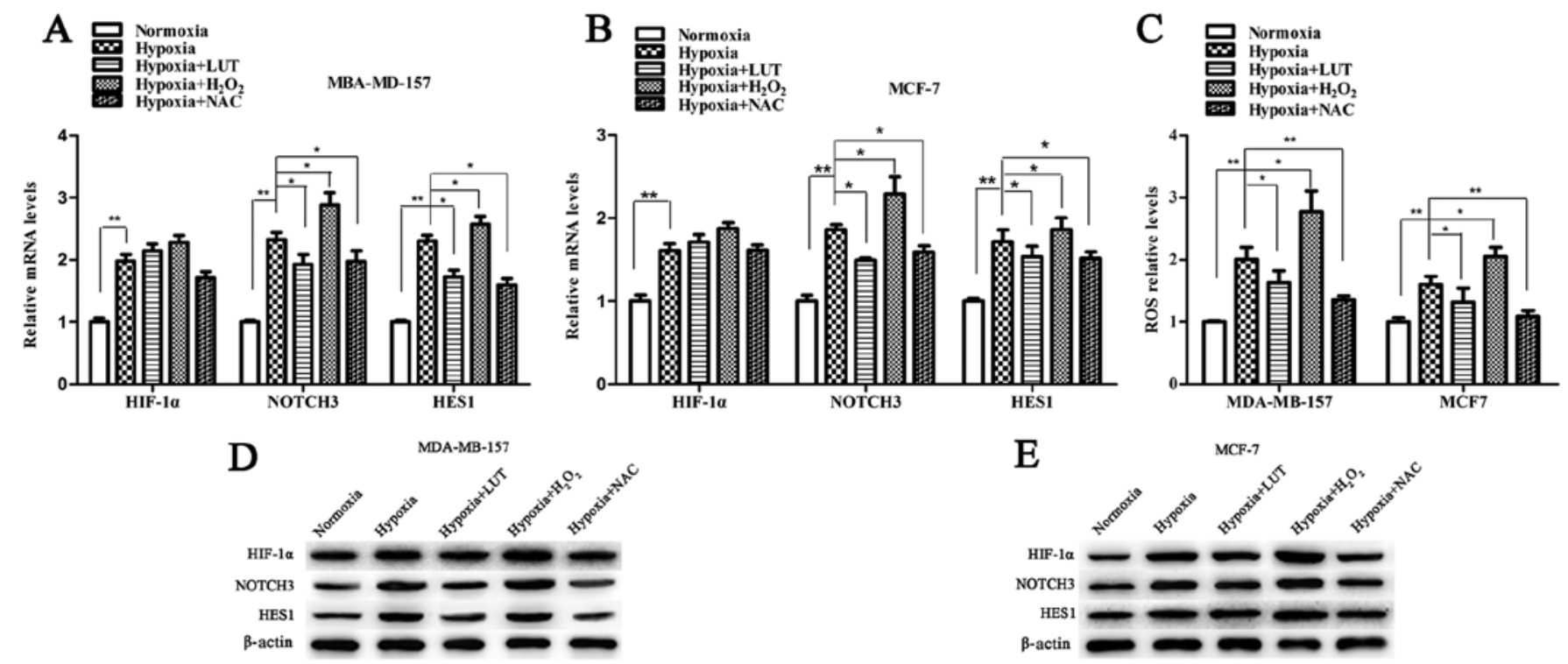

Figure 6. LUT suppresses the expression of HIF-1 $\alpha$ by decreasing the hypoxia-induced production of ROS in breast cancer cells. Reverse transcriptionquantitative polymerase chain reaction analysis demonstrating the relative mRNA levels of HIF-1 $\alpha$, NOTCH3 and HES1 in (A) MDA-MB-157 or (B) MCF-7 cells under the indicated treatment conditions for $24 \mathrm{~h}$. (C) Production of ROS in MDA-MB-157 or MCF-7 cells, measured by flow cytometry, under the indicated treatments for $24 \mathrm{~h}$. Western blot analysis of the expression of HIF-1 $\alpha$, NOTCH3 and HES1 in (D) MDA-MB-157 or (E) MCF-7 cells under the indicated treatments for $24 \mathrm{~h}$. The results were presented as the mean \pm standard deviation of triplicate experiments. " $\mathrm{P}<0.05$ and ${ }^{* *} \mathrm{P}<0.01$, with comparisons indicated by lines. LUT, lutein; HIF-1 $\alpha$, hypoxia inducible factor $1 \alpha$; ROS, reactive oxygen species; HES1, hairy and enhancer of split 1.

increased the expression levels of HIF-1 $\alpha$, NOTCH3, JAG1, JAG2, and DLL1, which were significantly accumulated in the two breast cancer cell lines compared with those under normoxia. Lutein decreased hypoxia-induced HIF-1 $\alpha$ protein expression, however, no significant difference in HIF- $1 \alpha$ mRNA expression was detected in MDA-MB-157 
and MCF-7 cells (Fig. 5A and B). This result indicated that lutein inhibited hypoxia-induced HIF-1 $\alpha$ protein expression in a post-transcriptional manner, and subsequently suppressed NOTCH receptors and ligands. These results revealed that lutein downregulated HES1 by suppressing hypoxia-induced HIF-1 $\alpha$ expression at the protein level, and by suppressing NOTCH signaling expression at the mRNA and protein levels.

Lutein inhibits the expression of HIF-1 $\alpha$ by decreasing the hypoxia-induced production of ROS in breast cancer cells. Previous studies have suggested that stabilization of HIF-1 $\alpha$ in low oxygen conditions is associated with an increase in ROS levels (26). To investigate whether lutein-induced downregulation of HIF-1 $\alpha$ and the NOTCH pathway in breast cancer cells under hypoxia is associated with ROS scavenging, the cells were treated under hypoxic conditions with $5 \mathrm{mM} \mathrm{H}_{2} \mathrm{O}_{2}$, $20 \mathrm{mM}$ NAC and $40 \mathrm{mM}$ lutein, respectively. The effect of hypoxia on intracellular ROS production was detected by flow cytometry. As presented in Fig. 6, cellular ROS levels under hypoxic conditions exposed to $\mathrm{H}_{2} \mathrm{O}_{2}$ were increased compared with those in the untreated control cells (Fig. 6C). Conversely, treatment with lutein and NAC (a known ROS scavenger) effectively reduced ROS levels in MDA-MB-157 and MCF-7 cells under hypoxia. Next, the association between the ROS accumulation and differential expression of HIF- $1 \alpha$, NOTCH3, and HES1 was determined using RT-qPCR and western blot analysis (Fig. 6). The mRNA levels of HIF-1 $\alpha$ were not significantly altered in response to ROS (Fig. 6A and B). The mRNA level of NOTCH3 and HES1 was significantly increased under hypoxia and $\mathrm{H}_{2} \mathrm{O}_{2}$ interference compared with the untreated control, but significantly decreased following treatment with lutein and NAC under hypoxia (Fig. 6A and B). Western blot analysis revealed that hypoxia and the $\mathrm{H}_{2} \mathrm{O}_{2}$-induced increase in cellular ROS levels significantly upregulated protein levels of HIF-1 $\alpha$, NOTCH components and HES1 compared with those in control cells. However, expression of these proteins was markedly suppressed following lutein and NAC treatment (Fig. 6D and E). Taken together, these results suggested that the increase of cellular ROS levels activated HIF- $1 \alpha$, as well as its downstream signaling under hypoxia, while lutein decreased the expression of HIF-1 $\alpha$ via the suppression of hypoxia-driven ROS in breast cancer cells.

\section{Discussion}

Due to improvements in screening, diagnostics, surgery and chemotherapeutic options, the mortality rate of breast cancer has abated over the last decade (7). However, metastatic breast cancer is heterogeneous and has a high mortality rate due to its inherently aggressive biology. Previous studies have determined that lutein suppresses multiple types of tumor, and the antitumor effects of lutein have been associated with a decline in proliferative signals and growth suppression $(21,22,27)$. Previous studies have focused on the associations between lutein and hypoxia. However, the mechanism underlying lutein-induced interference with the progression of breast cancer under hypoxic conditions remains unknown. The results of the present study confirmed that lutein was able to significantly inhibit the proliferation of human breast cancer cells in a concentration-dependent manner, by suppressing the proliferation, invasion, and migration of cells under hypoxia and normoxia.

It has previously been suggested that HES1 is a crucial transcription factor in cancer cells, and its upregulation is associated with cancer initiation, cell differentiation, tumor malignancy and tumorigenicity of cancer stem cells (13). These events contribute to the malignancy of cancer cells (28). The present study, consistent with previous studies $(14,24)$, demonstrated that HES1 expression levels increased in breast cancer cells under hypoxia compared with normoxia. Furthermore, hypoxia-induced HES1 accumulation was inhibited following lutein treatment, and lutein-induced decrement in HES1 expression was associated with the inhibition of cell proliferation under hypoxia. To confirm the involvement of HES1 in lutein-mediated antitumor effects under hypoxia, HES1 was overexpressed by transfecting breast cancer cells with HES1 overexpression constructs. Overexpression of HES1 reversed the antitumor effects of lutein.

Notably, HES1 has also been implicated in EMT-induced tumor invasion and metastasis, which facilitates the loss of cell adhesion and increases motility and survival in the detached condition $(17,29)$. A previous study has suggested that HES1 facilitates EMT processes by activating the protein kinase B/ phosphatase and tensin homolog axis, which has critical effects on the regulation of EMT in tumors (17). It is imperative to study the effects of lutein on EMT. Upregulation of Snail, Zinc finger E-box binding homeobox and Twist families, which are the direct transcriptional disruptors of E-cadherin (a marker of epithelial cells), and upregulation of mesenchymal molecular markers (including vimentin, $\mathrm{N}$-cadherin and fibronectin) are fundamental molecular events in EMT progression (30). The results of the present study indicated that lutein interference notably increased cell-cell interaction by upregulating E-cadherin, and maintained steady-state by downregulating $\mathrm{N}$-cadherin and vimentin in breast cancer cells. Therefore, these results indicated that lutein-induced inhibition of EMT is mediated by suppression of HES1. In addition, lutein-induced inhibition of cancer cell invasion and migration was observed, which was consistent with the EMT-associated features of cancer cells.

The regulation of HES1 is mediated by NOTCH signaling, which is a prominent canonical pathway that serves important functions in proliferation, invasion and apoptosis in cancer cells (31). Accumulating evidence indicates that phenotypes in malignant tumors are often accompanied by activated NOTCH signaling and elevated HES1, which are blocked by treatment with $\gamma$-secretase inhibitors (31). NOTCH intracellular domain (NICD) is released and translocated within the nucleus, where it facilitates transcription by binding with ligands of the NOTCH receptor (JAG1, JAG2 and DLL1, 3 and 4) (12). Previous studies have suggested a novel intersection between NOTCH signaling and HIF-1 $\alpha$. HIF- $1 \alpha$ induces the upregulation of the NOTCH ligand DLL4 gene, increases $\gamma$-secretase complex activity and mediates the stabilization of transcriptionally-active NICD (25). However, the mechanism underlying lutein-induced inhibition of HES1 remains to be fully understood. In the present study, HIF-1 $\alpha$ was observed to accumulate in breast cancer cells under hypoxia and to activate NOTCH signaling, which in turn induces the expression of the NOTCH target gene HES1 through NOTCH3 and NOTCH 
ligands in these cells. Meanwhile, treatment with lutein reversed hypoxia-induced upregulation of HIF-1 $\alpha$ and the NOTCH signaling pathway. Consequently, NOTCH-induced HES1 expression also decreased.

Increases in the rate of cellular metabolism and oxygen consumption have been attributed to hypoxic conditions in the center of breast cancer tissues, and these conditions exacerbate sprout angiogenesis and increase cancer invasiveness. ROS, including hydrogen peroxides, superoxide, hydroxyl radical and singlet oxygen, are chemically reactive molecules arising from miscellaneous enzymatic processes and the mitochondrial respiratory chain (32). Malignant cancer cells show elevated steady-state ROS stress compared with healthy cells, partly due to its involvement in tumorigenesis and oncogenic stimulation (11). Numerous oncogenic factors interact with high levels of ROS and promote the proliferation, invasion and metastasis of tumor cells. Previous studies have indicated that ROS are not only produced in hyperoxic conditions, but also under hypoxia (32). Hypoxia leads to an increase in the production of ROS from different sources, including mitochondrial transport of electrons, NADPH oxidase and xanthine oxidase. ROS are involved in diverse biological and pathological activities. ROS levels are frequently high in cancer cells and regulate cancer cell proliferation, invasion, and apoptosis $(33,34)$. Moderate ROS levels activate a cascade of signals as a secondary messenger, and regulate responses to various stimulations (33). Previous studies have demonstrated that ROS serve an important modulatory function in the mediation of HIF- $1 \alpha$ activity under hypoxia in vivo as well as in vitro (11). ROS production is required for the direct or indirect regulation of translocation, activation, and degradation of HIF- $1 \alpha$ via PHD family modification in response to hypoxia (9). To confirm whether lutein suppresses hypoxia-induced HIF-1 $\alpha$ as well as its downstream pathways, NOTCH signaling and HES1, our group also evaluated the effect of NAC and $\mathrm{H}_{2} \mathrm{O}_{2}$ in breast cancer cells under hypoxia. The results indicated that $\mathrm{H}_{2} \mathrm{O}_{2}$ exposure was responsible for the elevated ROS levels and increased HIF-1 $\alpha$ expression, which subsequently increased the expression of NOTCH signaling molecules and HES1, while the effect of NAC was the opposite. These results suggested that, compared with NAC, the effect of luteininduced HIF-1 $\alpha$ expression is attributable to the reduction of ROS under hypoxia; as this effect was reversible when ROS levels were elevated by $\mathrm{H}_{2} \mathrm{O}_{2}$.

In conclusion, the results of the present study demonstrated that lutein suppressed hypoxia-induced proliferation, invasion, and migration of breast cancer cells in vitro by decreasing HES1 expression. Lutein markedly suppressed hypoxiainduced ROS levels, which is essential for the stability of HIF-1 $\alpha$. Subsequently, lutein inhibited HES1 expression through the NOTCH signaling pathway in breast cancer cells. Furthermore, lutein-induced HES1 inhibition contributed to inhibition of EMT, a process in various malignant tumors that is associated with increased invasion and migration. These results provide insight into the molecular mechanisms underlying the regulation of HES1 by lutein through HIF-1 $\alpha$ and NOTCH signaling, suggesting that lutein may be used as a potential agent for the development of novel therapeutic approaches for the treatment of breast cancer in the future.

\section{Acknowledgements}

Not applicable.

\section{Funding}

The present study was supported by grants from the National Natural Science Foundation of China (grant no. 81500433) and the Foundation of Henan Educational Committee (grant no. 16A310003)

\section{Availability of data and materials}

The datasets generated during the current study are available from the corresponding author on reasonable request.

\section{Authors' contributions}

SZ and MW conceived the study and participated in its design and coordination. YL and XL performed the experiments and contributed to data collection. YL and YZ analyzed the data and drafted the manuscript. PW and JY assisted in designing experiments and provided technical expertise in conducting experiments, and reviewed the manuscript. All authors read and approved the final manuscript.

\section{Ethics approval and consent to participate}

Not applicable.

\section{Consent for publication}

Not applicable.

\section{Competing interests}

The authors declare that they have no competing interests.

\section{References}

1. Harbeck N and Gnant M: Breast cancer. Lancet 389: 1134-1150, 2017.

2. Fan L, Strasser-Weippl K, Li JJ, St Louis J, Finkelstein DM, Yu KD, Chen WQ, Shao ZM and Goss PE: Breast cancer in China. Lancet Oncol 15: e279-e289, 2014.

3. Sariego J: Breast cancer in the young patient. Am Surg 76: 1397-1400, 2010.

4. Fillmore CM and Kuperwasser C: Human breast cancer cell lines contain stem-like cells that self-renew, give rise to phenotypically diverse progeny and survive chemotherapy. Breast Cancer Res 10: R25, 2008.

5. Mayer IA, Abramson VG, Lehmann BD and Pietenpol JA: New strategies for triple-negative breast cancer - deciphering the heterogeneity. Clin Cancer Res 20: 782-790, 2014.

6. Ward C, Langdon SP, Mullen P, Harris AL, Harrison DJ, Supuran CT and Kunkler IH: New strategies for targeting the hypoxic tumour microenvironment in breast cancer. Cancer Treat Rev 39: 171-179, 2013.

7. Tang Y, Wang Y, Kiani MF and Wang B: Classification, treatment strategy, and associated drug resistance in breast cancer. Clin Breast Cancer 16: 335-343, 2016

8. Semenza GL: Targeting HIF-1 for cancer therapy. Nat Rev Cancer 3: 721-732, 2003.

9. Zepeda AB, Pessoa A Jr, Castillo RL, Figueroa CA, Pulgar VM and Farías JG: Cellular and molecular mechanisms in the hypoxic tissue: Role of HIF-1 and ROS. Cell Biochem Funct 31: 451-459, 2013. 
10. Balamurugan K: HIF-1 at the crossroads of hypoxia, inflammation, and cancer. Int J Cancer 138: 1058-1066, 2016.

11. Galanis A, Pappa A, Giannakakis A, Lanitis E, Dangaj D and Sandaltzopoulos R: Reactive oxygen species and HIF-1 signalling in cancer. Cancer Lett 266: 12-20, 2008.

12. Liu ZH, Dai XM and Du B: Hes1: A key role in stemness, metastasis and multidrug resistance. Cancer Biol Ther 16: 353-359, 2015.

13. Rani A, Greenlaw R, Smith RA and Galustian C: HES1 in immunity and cancer. Cytokine Growth Factor Rev 30: 113-117, 2016.

14. Danza G, Di Serio C, Rosati F, Lonetto G, Sturli N, Kacer D, Pennella A, Ventimiglia G, Barucci R, Piscazzi A, et al: Notch signaling modulates hypoxia-induced neuroendocrine differentiation of human prostate cancer cells. Mol Cancer Res 10: 230-238, 2012.

15. Liu J, Lu WG, Ye F, Cheng XD, Hong D, Hu Y, Chen HZ and Xie X: Hes1/Hes5 gene inhibits differentiation via down-regulating Hash 1 and promotes proliferation in cervical carcinoma cells. Int J Gynecol Cancer 20: 1109-1116, 2010.

16. Yuan R, Ke J, Sun L, He Z, Zou Y, He X, Chen Y, Wu X, Cai Z, Wang L, et al: HES1 promotes metastasis and predicts poor survival in patients with colorectal cancer. Clin Exp Metastasis 32: 169-179, 2015

17. Wang SC, Lin XL, Wang HY, Qin YJ, Chen L, Li J, Jia JS, Shen HF, Yang S, Xie RY, et al: Hes1 triggers epithelial-mesenchymal transition (EMT)-like cellular marker alterations and promotes invasion and metastasis of nasopharyngeal carcinoma by activating the PTEN/AKT pathway. Oncotarget 6: 3671336730, 2015.

18. Johnson EJ: A biological role of lutein. Food Rev Int 20: 1-16, 2004.

19. Nwachukwu ID, Udenigwe CC and Aluko RE: Lutein and zeaxanthin: Production technology, bioavailability, mechanisms of action, visual function, and health claim status. Trends Food Sci Technol 49: 74-84, 2016.

20. Sindhu ER, Firdous AP, Ramnath V and Kuttan R: Effect of carotenoid lutein on $\mathrm{N}$-nitrosodiethylamine-induced hepatocellular carcinoma and its mechanism of action. Eur J Cancer Prev 22: 320-327, 2013

21. Lakshminarayana R, Sathish UV, Dharmesh SM and Baskaran V: Antioxidant and cytotoxic effect of oxidized lutein in human cervical carcinoma cells (HeLa). Food Chem Toxicol 48: 1811-1816, 2010.

22. Rafi MM, Kanakasabai S, Gokarn SV, Krueger EG and Bright JJ: Dietary lutein modulates growth and survival genes in prostate cancer cells. J Med Food 18: 173-181, 2015.
23. Livak KJ and Schmittgen TD: Analysis of relative gene expression data using real-time quantitative PCR and the 2(-Delta Delta C(T)) Method. Methods 25: 402-408, 2001.

24. Chen J, Imanaka N, Chen J and Griffin JD: Hypoxia potentiates Notch signaling in breast cancer leading to decreased E-cadherin expression and increased cell migration and invasion. Br J Cancer 102: 351-360, 2010.

25. Borggrefe T, Lauth M, Zwijsen A, Huylebroeck D, Oswald F and Giaimo BD: The Notch intracellular domain integrates signals from Wnt, Hedgehog, TGF//BMP and hypoxia pathways. Biochim Biophys Acta 1863: 303-313, 2016.

26. Wang Y, Ma J, Shen H, Wang C, Sun Y, Howell SB and Lin X: Reactive oxygen species promote ovarian cancer progression via the HIF-1 $\alpha /$ LOX/E-cadherin pathway. Oncol Rep 32: 2150-2158, 2014.

27. Reynoso-Camacho R, González-Jasso E, Ferriz-Martínez R, Villalón-Corona B, Loarca-Piña GF, Salgado LM and Ramos-Gomez M: Dietary supplementation of lutein reduces colon carcinogenesis in DMH-treated rats by modulating K-ras, PKB, and $\beta$-catenin proteins. Nutr Cancer 63: 39-45, 2011.

28. Sang L, Roberts JM and Coller HA: Hijacking HES1: How tumors co-opt the anti-differentiation strategies of quiescent cells. Trends Mol Med 16: 17-26, 2010.

29. Gao F, Huang W, Zhang Y, Tang S, Zheng L, Ma F, Wang Y, Tang $\mathrm{H}$ and $\mathrm{Li} \mathrm{X}$ : Hes1 promotes cell proliferation and migration by activating Bmi-1 and PTEN/Akt/GSK3 $\beta$ pathway in human colon cancer. Oncotarget 6: 38667-38680, 2015 .

30. Yilmaz M and Christofori G: EMT, the cytoskeleton, and cancer cell invasion. Cancer Metastasis Rev 28: 15-33, 2009.

31. Wong NKY, Fuller M, Sung S, Wong F and Karsan A Heterogeneity of breast cancer stem cells as evidenced with Notch-dependent and Notch-independent populations. Cancer Med 1: 105-113, 2012.

32. Movafagh S, Crook S and Vo K: Regulation of hypoxia-inducible factor-la by reactive oxygen species: New developments in an old debate. J Cell Biochem 116: 696-703, 2015.

33. Zhang C, Cao S, Toole BP and Xu Y: Cancer may be a pathway to cell survival under persistent hypoxia and elevated ROS: A model for solid-cancer initiation and early development. Int J Cancer 136: 2001-2011, 2015.

34. Cao L, Chen X, Xiao X, Ma Q and Li W: Resveratrol inhibits hyperglycemia-driven ROS-induced invasion and migration of pancreatic cancer cells via suppression of the ERK and p38 MAPK signaling pathways. Int J Oncol 49: 735-743, 2016. 